\title{
«L'EIL ÉLECTRIQUE » ET « LA TORPILLE VOLANTE » : PISTES POUR UNE HISTOIRE DU DRONE À PARTIR DE L'HISTOIRE TÉLÉVISUELLE
}

\author{
Anne-Katrin Weber
}

BSN Press | « A contrario »

2019/2 n² 29 | pages 81 à 98

ISSN $1660-7880$

ISBN 9782940648115

Article disponible en ligne à l'adresse :

https://www.cairn.info/revue-a-contrario-2019-2-page-81.htm

Distribution électronique Cairn.info pour BSN Press.

(C) BSN Press. Tous droits réservés pour tous pays.

La reproduction ou représentation de cet article, notamment par photocopie, n'est autorisée que dans les limites des conditions générales d'utilisation du site ou, le cas échéant, des conditions générales de la licence souscrite par votre établissement. Toute autre reproduction ou représentation, en tout ou partie, sous quelque forme et de quelque manière que ce soit, est interdite sauf accord préalable et écrit de l'éditeur, en dehors des cas prévus par la législation en vigueur en France. Il est précisé que son stockage dans une base de données est également interdit. 


\title{
«L'œil électrique » et «la torpille volante»: pistes pour une histoire du drone à partir de l'histoire télévisuelle
}

\author{
ANNE-KATRIN WEBER
}

\begin{abstract}
Il convient de rendre hommage aux scientifiques et aux ingénieurs dont la recherche et la force d'innovation, les connaissances techniques et l'ingéniosité ont fait de la télévision aérienne une arme de guerre efficace, et qui ont ouvert la voie à des progrès monumentaux en permettant d'étendre les champs d'application de la télévision en temps de paix.
\end{abstract}

(Sarnoff 1946: 292)

D ans l'introduction au numéro de septembre 1946 de la RCA Review, le journal technique de sa société, David Sarnoff passe en revue plus d'une décennie de recherches militaires dans le domaine télévisuel. Selon lui, les investigations sur la airborne televison ("télévision aéroportée») ${ }^{1}$ au sein de la Radio Corporation of America ont débuté en 1934 lorsque Vladimir K. Zworykin, ingénieur en chef et pionnier du télévisuel, écrit un premier mémorandum sur le sujet, imprimé dans le numéro de la RCA Review. Intitulé Flying Torpedo with an Electric Eye, le texte de Zworykin prévoit deux fonctions principales pour la télévision militaire. Elle doit premièrement fonctionner comme un appareil auxiliaire à la gestion à distance d'armes semi-automatisées. La caméra intégrée dans un missile peut ainsi surveiller « l'état des commandes de la torpille, la bonne réception des signaux de commande, l'altitude, etc.» (Zworykin 1946 [1934] : 297) et donc transmettre aux pilotes des informations sur l'état de l'équipement. Deuxièmement, plus qu'une simple prothèse de la vision humaine, la télévision se fait létale: en transmettant des images directement depuis le nez du missile, la télévision fait voir à «l'opérateur [...] la cible à travers "l'œil” de la torpille à l'approche de sa marque » (Zworykin 1946 [1934] : 294). Grâce à la vision à distance, l'œil du pilote et l'œil du missile ne font qu'un : la «torpille volante avec un œil électrique » fait de "voir» et «tuer " des synonymes (Fig. 1).

1 Sauf mention contraire, les traductions sont de l'auteure. 

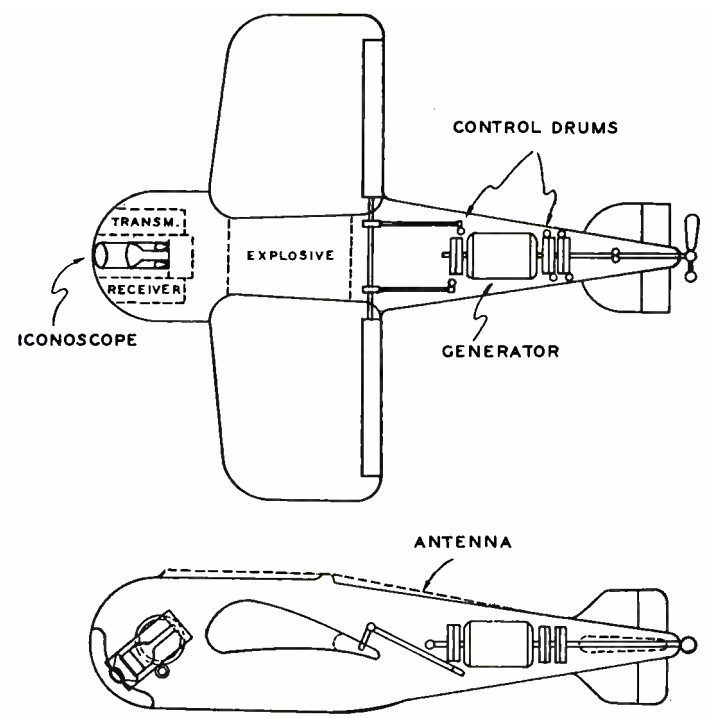

Fig. 1-The Flying Torpedo.

Fig. 1: La «torpille volante avec un ceil electrique» : esquisse pour un missile téléguidé intégrant un système télévisuel, proposée en 1934 par Zworykin, directeur des recherches télévisuelles à la RCA. (Source: Zworykin 1946 [1934] : 296.)

Larticle de Zworykin de 1934 et sa publication en 1946 témoignent du dynamisme des recherches télévisuelles menées dès l'entre-deux-guerres: alors que la RCA se profile durant les années 1930 grâce au développement de la télévision commerciale - lancée en 1939 à la New York World's Fair -, elle poursuit en parallèle des recherches sur d'autres applications possibles du média, notamment dans le domaine militaire. Les numéros de la RCA Review de septembre et décembre 1946 font justement état de ces nombreuses études, tenues en partie secrètes durant la guerre. Ils réunissent des résumés (toujours très techniques) de différents projets. Les articles incluent notamment une discussion sur les "équipements télévisuels pour les avions", la présentation d'un "système de reconnaissance télévisuelle pour avion » ou encore la description d'un "équipement miniature de télévision " destiné à être placé dans un missile. Publié en même temps que des recherches plus récentes, le texte de Zworykin de 1934 constitue l'étape pionnière d'un effort plus global, celui du soutien de la RCA aux forces armées américaines dans le domaine des engins téléguidés.

La fin du conflit mondial n'est toutefois pas seulement l'occasion d'énumérer les accomplissements réalisés; elle présente également un moment clé pour le réajustement de la recherche et de la production. À cet égard, le transfert des savoirs et des 
technologies de la recherche militaire vers le civil est primordial, comme le laisse entendre Sarnoff dans son introduction. Dans ses remerciements aux ingénieurs pour leurs efforts durant la guerre, il établit un lien direct entre les travaux réalisés et ceux à venir. Selon lui, son équipe a « ouvert la voie à des progrès monumentaux étendant les champs d'application de la télévision en temps de paix» (Sarnoff 1946: 292).

La recherche télévisuelle qui a été effectuée pour remplir des objectifs militaires n'est en effet pas perdue à la fin du conflit puisque différents composants et systèmes technologiques seront adaptés à des usages commerciaux. À ce titre, le tube cathodique de type Image Orthicon, utilisé dès 1944 dans des missiles téléguidés, constitue l'exemple le plus parlant. Le tube Image Orthicon se caractérise par sa haute sensibilité qui le rend performant même dans des conditions d'éclairage médiocres. Cette sensibilité accrue le distingue des tubes cathodiques précédents qui nécessitent un éclairage de studio puissant (Roe 1950). Selon l'historien de la télévision Albert Abramson, les caméras équipées d'un tube Image Orthicon facilitent en effet l'essor de la télévision en tant que mass media dans l'après-guerre. Légères et transportables, elles ne requièrent pas d'équipement d'éclairage spécifique et peuvent plus facilement quitter les studios. Pour Abramson, “[l’]introduction de cette caméra a rendu la télévision véritablement praticable» (Abramson 2003:18).

Si la recherche militaire nourrit ainsi directement l'émergence de la télévision commerciale, elle est aussi à l'origine d'un nouveau champ télévisuel qui prend son essor dès les années 1940. Lorsque Sarnoff célèbre le progrès réalisé par les ingénieurs de la RCA qui ont permis "d'étendre les champs d’application de la télévision ", il fait également allusion à ce qu'on labellisera "télévision industrielle» ou «télévision en circuit fermé». En mettant à profit les dispositifs télévisuels militaires dans le cadre industriel, ces applications visent à rendre le média utile à des fins de contrôle, de surveillance, et de gestion de divers processus de manufacture et de manutention.

Partant du mémorandum de Zworykin, cet article propose d'étudier l'histoire des drones par le biais de l'histoire de la télévision. S’intéressant plus précisément aux échanges entre les domaines militaire et civil, sa focale s'éloigne d'une étude stricte des drones pour embrasser une approche d'histoire des médias et d'archéologie télé-

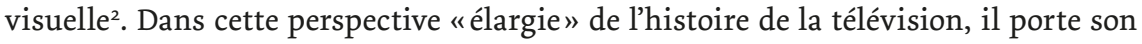
attention sur les dispositifs télévisuels non institutionnels; ceux-ci ne participent pas

2 Sur la notion d'archeologie des médias, voir Parikka 2012 ; Fickers \& Weber 2015 discutent la notion d'archéologie télévisuelle. 
à la définition du mass media diffusant un programme régulier dans les espaces domestiques, mais sont du ressort de domaines relativement négligés par l'historiographie, à savoir le militaire et l'industrie. Le double déplacement - des drones vers la télévision et de la télévision institutionnelle vers la télévision militaire et industrielle - permet de cartographier la circulation des pratiques et des techniques et de souligner la perméabilité des champs militaire et civil, souvent séparés au sein du débat actuel.

Larticle souhaite en particulier réfléchir à deux aspects centraux du dispositif dronique qui sont directement liés à l'utilisation de composants télévisuels. Techno-

84 logie de la vision à distance, la télévision est centrale pour la réalisation d'un engin "sans équipage" puisqu'elle retire le corps du soldat du champ de bataille et le protège derrière l'écran. Cette mise à distance du corps humain s'opère aussi sur le plan discursif. Elle se retrouve dans les descriptions des machines "sans hommes", qui seront reprises dans le champ industriel sous l'étiquette de l'automation. Le « unmanning " 3 du drone que permettent les systèmes télévisuels est ainsi emblématique de la circulation de concepts et d'imaginaires entre les domaines militaires et industriels.

Lanalyse de la fonctionnalité de l'image télévisuelle dans des environnements techniques militaro-industriels offre une autre manière de penser les liens entre des pratiques militaires et industrielles. Pour cela, l'article recourt à la notion d'، images opérationnelles" (Farocki 2004) qui permet d’appréhender les images télévisuelles non pas en tant que représentations mais en tant qu'instruments. Lorsque voir signifie faire, la destruction et la production deviennent des opérations qui s'apparentent (Farocki dans Pantenburg 2017: 55).

\section{Drone-cible, drone d'assaut : une brève histoire}

Durant la seconde moitié des années 1930, plusieurs services de télévision réguliers apparaissent et la commercialisation de la télévision en tant que mass media national et domestique se prépare en Europe et aux États-Unis (Abramson 1987; Burns 1998). En parallèle à cette première phase d'institutionnalisation, de multiples formes télévisuelles sont développées. Du grand écran au visiophone, la télévision est conçue comme un média qui s'insère dans des espaces diversifiés et qui est capable de s'adapter à des formes communicationnelles multiples. Le "torpédo volant» muni d'un système télévisuel tel qu'esquissé par Zworykin constitue dès lors une preuve supplémentaire de lélasticité

3 En anglais, les drones s'appellent aussi les Unmanned Aerial Vehicle (UAV). J'emprunte le terme unmanning des travaux de Katherine Chandler qui analyse l'histoire des drones sous l'angle de cette notion désignant l'action de se défaire de l'humain (Chandler 2020). 
du média dont l'identité est particulièrement flexible. L'arme télévisuelle de 1934 n'est que l'un des résultats d'une longue liste de recherches qui explorent le maximum d'applications possibles pour la télévision dans des secteurs extrêmement variés (cadre domestique; divertissement collectif; télécommunications; applications militaires) (Uricchio 2009; Keilbach \& Stauff 2013; Weber à paraître).

Part intégrante de l'histoire de la télévision, le mémorandum de 1934 s’inscrit également dans l'histoire militaire, et plus particulièrement dans l'histoire longue du drone en tant qu'engin volant sans équipage. Cette histoire remonte au moins aux années 1910 et aux premières expériences réalisées par des ingénieurs américains et britanniques (Zubeldia 2012; Everett 2015; Hall Kindervater 2016). Contrairement aux drones contemporains, qui lâchent leur charge explosive quand ils arrivent à destination puis retournent à la base militaire, ces premiers engins fonctionnent avant tout comme des bombes volantes. Ils s'apparentent ainsi aux missiles de croisière - des missiles à longue portée - qui atteignent leur cible en suivant une trajectoire désignée d'avance. Par exemple, le Kettering Bug, construit en bois et muni d'ailes de mousseline et de papier, est conçu comme un projectile autonome qu'on lance depuis un charriot sur rails. La Curtiss-Sperry «Flying Bomb», également développée durant la Première Guerre aux États-Unis, est quant à elle catapultée dans les airs pour y voler un certain temps avant que son moteur ne s'arrête automatiquement et qu'elle tombe sur la cible pour la détruire en explosant (Packer \& Reeves 2013 : 320-321; Everett 2015: 248-260).

C’est en 1936 que le terme de «drone» aurait été proposé pour la première fois par l'américain Delmar Fahrney (Howeth 1963: 481; pour une discussion de la paternité du terme, voir Everett 2015 : 306). Ingénieur formé au Massachusetts Institute of Technology (MIT), Fahrney est mandaté au milieu des années 1930 par la US Navy pour monter un programme de recherche portant sur des avions sans équipage et télécommandés. Ce projet est initié suite à la Second London Naval Disarmament Conference de 1936, lorsque les officiers de la marine américaine découvrent les avancées britanniques dans le domaine des avions sans équipage (Everett 2016 : 306). À l'instar de l'appareil britannique "Queen Bee», qui est aujourd'hui volontiers présenté comme "la mère de tous les drones " ${ }^{4}$, les premiers aéronefs sans équipage américains sont conçus pour l'entraînement des artilleurs anti-aériens. Ils servent à imiter une attaque aérienne et endossent donc le rôle de cible à abattre par les soldats apprentis. Le fonctionnement basique d'un tel drone d'entraînement requiert un opérateur au sol qui contrôle

4 Voir par exemple: http://www.vintagewings.ca/VintageNews/Stories/tabid/116/articleType/ArticleView/ articleId/484/The-Mother-of-All-Drones.aspx, consulté le 13 novembre 2019. 
l'engin lors du décollage et un pilote dans un avion qui suit le drone et le conduit à distance jusqu'à ce que ce dernier soit touché (Fahrney 1980). Le premier usage des drones au sein des forces armées est donc défensif: se muant en missiles à abattre, ils font partie des stratégies développées pour contrer les attaques par les airs.

Les expériences de "torpilles volantes" menées pendant la Première Guerre mondiale connaissent un regain d'intérêt à la fin des années 1930, lorsque les drones d'assaut viennent s'ajouter à la liste des nouvelles armes (Chandler 2017: 95). Les réflexions de Zworykin à ce sujet prennent notamment forme dans le cadre de la collaboration 86 étroite entre la RCA, le National Defense Research Committee (NDRC) et le Office of Scientific Research and Development (OSRD). L'OSRD est établi par le gouvernement Roosevelt pour surveiller et gérer la recherche scientifique dans les universités et les laboratoires des entreprises privées dès $1941^{5}$. Dirigé par Vannevar Bush, cet organe s'ajoute au NDRC, créé sous impulsion de Bush une année plus tôt, lorsque cette première structure, focalisée sur la recherche, s'avère trop limitée pour remplir les objectifs de guerre. Dans le cadre de l'OSRD, industrie et science collaborent désormais étroitement avec le secteur militaire pour mettre au point des systèmes d'armement (Edwards 1996: 46). Concentrant les efforts de guerre, l'OSRD devient l'incubateur de nombreux mégaprojets, dont le plus connu est le projet « Manhattan». Ce projet servira de cadre pour le développement de la bombe atomique; celle-ci fournira une première opportunité pour tester le transfert de la télévision militaire dans un cadre industriel.

\section{Mettre à distance}

Préserver le pilote de tout danger physique est l'objectif principal qui sous-tend le développement des drones d'assaut, destinés au champ de bataille. L'avantage de ce dispositif automatisé est en effet d'" exfiltrer» du lieu des combats le corps du soldat qui devient dès lors intouchable. Dans sa Théorie du drone, Grégoire Chamayou identifie justement cette "topographie spécifique» comme l'un des fondements de la guerre asymétrique par armes téléguidées. Elle se caractérise par le fait qu'« on y meurt encore, mais d’un côté seulement" (Chamayou 2013: 40). En tant que média de la vision à distance, la télévision fournit la technologie nécessaire à la concrétisation de cette topographie spécifique. Intégrée à des systèmes d'armement, elle éloigne d'une part les corps du champ de bataille, mais elle permet d'autre part de rapprocher et rendre visible la zone de combat. En effet, comme le souligne Antoine Bousquet dans son histoire de la perception

5 A ce sujet soulignons qu'outre la RCA, d'autres entreprises et laboratoires, tels que la Farnsworth Television and Radio Corporation, sont soutenus par des ressources publiques pour faire avancer la recherche sur la télévision à des fins militaires. 
militaire, la télévision permet « une libération définitive de l'œil humain de sa coquille corporelle» (Bousquet 2018: 62).

Zworykin rappelle lui-même à plusieurs reprises l'importance de mettre les pilotes à l'abri du feu ennemi grâce à la télévision:

"la torpille peut être guidée vers sa cible par une commande radio à ondes courtes, l'opérateur pouvant voir la cible à travers "l'œil" de la torpille à mesure que cette première s'approche. L'avion porteur reçoit l'image vue par la torpille tout en restant à une altitude qui lui assure d'être hors de portée de l'artillerie.» (Zworykin 1946 [1934]: 294)

Au milieu des années 1930, il est évident que l'aviation militaire a profondément transformé la manière de mener une guerre: elle a étendu le champ de bataille à l'«espace vertical» (Parks dans ce numéro) et a permis aux armées de bénéficier du regard englobant de la vue aérienne sur le territoire ennemi (Hippler 2014). Pouvoir soustraire les pilotes de chasse des feux ennemis est alors un argument de poids en faveur des drones. Dans son article, Zworykin évoque cependant encore une autre raison qui l'aurait poussé à penser son arme. L’ingénieur la conçoit en effet comme une réponse concrète aux missions-suicide des troupes japonaises qui, en 1934 déjà, auraient organisé « un régiment suicidaire pour contrôler les torpilles de surface et les torpilles aériennes » (Zworykin 1946 [1934]: 294). Face au défi soulevé par l'ennemi, taxé de barbarie à cause de l'usage de ces soldats suicidaires, la télévision se conçoit comme une solution technologique "supérieure», civilisée et rationnelle (Chandler 2017). À l'emploi de la force brute (allant jusqu'à l'auto-annihilation), Zworykin oppose une force technologique que matérialise la technologie télécommunicationnelle. L'argument de la sécurité du personnel américain est ainsi associé à l'affirmation de la supériorité (technologique mais aussi raciale) d'une nation sur l'autre.

En 1934, Zworykin décrit dès lors ce qui deviendra une caractéristique primordiale de la guerre des drones post-9/11. Préservés par la distance, les soldats des guerres droniques contemporaines ne font pas face au duel des combattants. Ils interviennent depuis leurs espaces sanctuarisés, situés à des milliers de kilomètres des espaces vitaux des communautés visées. Le dispositif du drone contemporain - qui contient des composants télévisuels - se base sur une interprétation asymétrique de la valeur d'une vie humaine que résume l'idée d'une guerre à «zéro morts». Selon Grégoire Chamayou, il traduit un «nationalisme de l'autopréservation vitale» qui témoigne d'une conception profondément impérialiste des conflits internationaux contemporains (Chamayou 2013: 189). 
Cette asymétrie revendiquée par les gouvernements employant les drones armés, fonde la «nécroéthique du drone» en tant qu'éthique du «bien tuer» (2013: 206).

\section{Se défaire de l'homme}

Dans les sources citées, la mise à distance géographique de la guerre et la préservation des pilotes sont réalisées technologiquement grâce à la télévision. Sur le plan discursif, elles se traduisent en premier lieu par l'accent mis sur l'autonomie des systèmes technologiques. Les armes téléguidées sont en effet souvent décrites sans que soient mentionnés les soldats qui les manipulent. Dans sa discussion du mémorandum de 1934, la 88 politologue Katherine Chandler soulève l'utilisation récurrente du passif pour expliquer les fonctions de la torpille de Zworykin. Selon Chandler, Zworykin élude de la sorte les interactions entre le pilote et sa machine pour se focaliser sur celles entre l'avion et la télévision. Cette opération rhétorique, au cœur de la description de Zworykin, se défait ainsi de l'humain : l'arme téléguidée est conceptualisée comme une arme (quasi) autonome (Chandler 2017; Chandler 2020). Cette occultation de l'intervention humaine n'est pas propre à la note de l'ingénieur; elle se retrouve dans de nombreux textes sur les armes téléguidées. Elle est particulièrement saillante dans un rapport de la RCA paru après-guerre, où le drone est « humanisé» en devenant un acteur à part entière:

"[...] l'avion aussi appelé drone. Celui-ci pourrait être un petit avion construit à moindre coût, conçu comme un avion consomptible lors de sa première mission, à l'instar des nombreux avions suicidaires japonais. En réalité, il s'agissait généralement d’un appareil inutilisé et obsolète [...] devenu superflu pour tout autre service. Dans son nez, un équipement télévisuel était installé. Celui-ci transmettait sur un écran dans la régie [se trouvant] sur un avion mère sur terre ou dans les airs - généralement dans les airs afin de maintenir le contact. L'avion mère pouvait naviguer très haut dans la stratosphère pendant que ses charges sans équipage ["manless charge"] plongeaient vers leurs cibles. [...] Si la cible esquivait, le drone pouvait changer de cap et poursuivre la poursuite. Tel était le plan d'action. Pour rappel, le drone télévisé [sic] n'est jamais entré en combat. Des essais ont cependant été effectués avec succès, le drone étant contrôlé depuis des stations terrestres, des navires [...] et enfin des avions en altitude. Bien que des diffcultés aient été rencontrées tout au long des expériences, les composants télévisuels ont été achevés et le drone aurait bientôt pu être utilisé dans les eaux japonaises si la bombe atomique n'avait pas mis fin à la guerre à l'été 1945.» ("Radio Research in Wartime»)

Ce passage mérite d'être cité en entier car il constitue un exemple emblématique de la manière dont les discours masquent le poids de l'humain dans les systèmes 
technologiques. Ici, même la bombe atomique, dont le développement a mobilisé des milliers, voire des centaines de milliers de personnes, semble littéralement tomber du ciel toute seule. La présence humaine se limite à la mention de la manless charge qui fait référence à l’homme uniquement par la négation.

Léviction de l'humain se réalise également dans les représentations visuelles produites par les drones. Les images tests des expériences menées par le NDRC au début des années 1940 enregistrent des paysages vides de toute trace humaine: la faible qualité de l'image télévisuelle ainsi que les conditions d'expérimentations transforment la surface de la Terre en plan d'inscription pour la technologie testée (voir aussi Parks dans ce numéro). De telles images anticipent ainsi ce que le cinéaste Harun Farocki observera à propos des images de la Guerre du Golfe, souvent qualifiée de première guerre diffusée en direct sur les postes télévisuels (Rollins 1992). Les networks américains et autres télévisions privées et publiques transmettaient alors des images prises depuis des bombes intelligentes. Pour Farocki, dans ces images d'une guerre téléguidée et automatisée, le champ de bataille est «inhabité » (Farocki 2004:15) (Fig. 2).

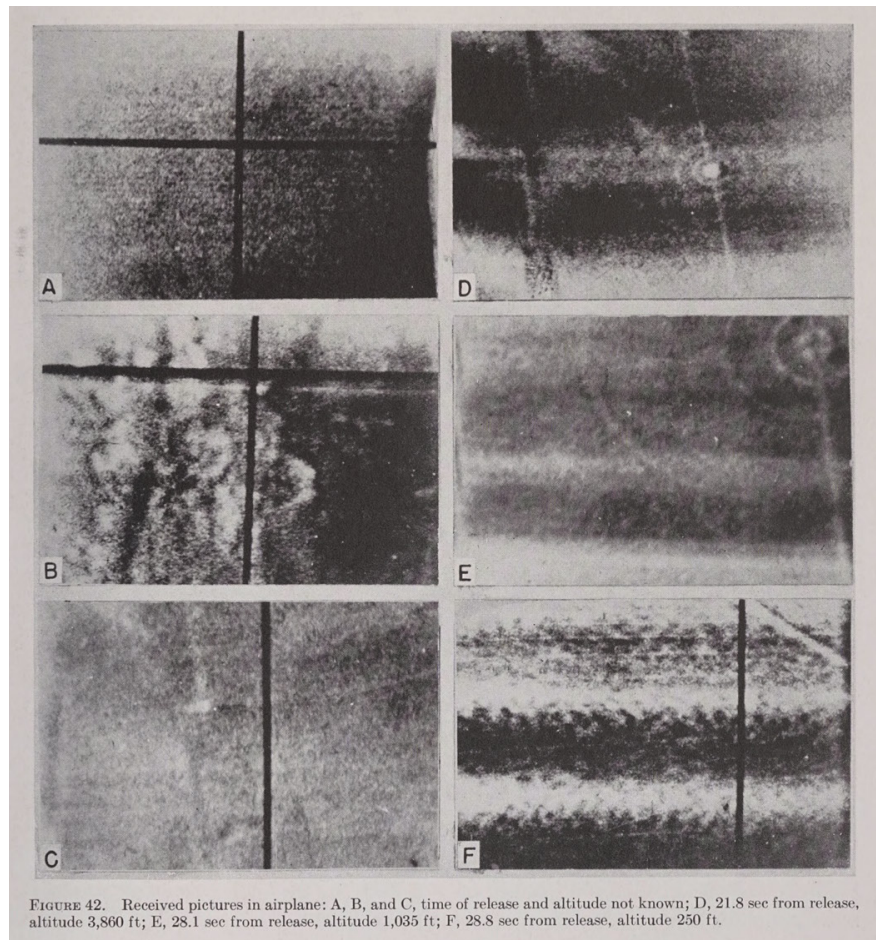

Fig. 2: Vues telévisuelles transmises depuis les missiles et bombes téléguidés.

(Source: NDRC 1946: 125.) 


\section{Du militaire à l'industrie : la télévision en circuit fermé}

Alors que l'éviction de l'homme de la machine est un objectif déclaré des discours sur les drones dans les années 1940, l'opération des engins requiert une intervention massive de la part d'acteurs humains. Cela est d'autant plus le cas qu'ils demeurent des dispositifs expérimentaux affichant d'importants problèmes sur le plan technique (Chandler 2017). En effet, comme le laisse entendre le rapport cité plus haut, en termes d'objectifs militaires, la recherche sur les drones n'est pas couronnée de grands succès. Les comptes-rendus rédigés dans l'immédiat après-guerre mettent en avant les nombreuses difficultés rencontrées dans le perfectionnement de ces engins

90 et concluent qu' " aucun système de missile télévisuel n’a totalement réussi au moment où la Deuxième Guerre mondiale se termine" (NDRC 1946: 4). De manière similaire, la littérature secondaire s'accorde pour qualifier les tests dans le domaine de la télévision militaire d'échecs qui n'ont pas eu d'effets marquants sur le déroulement du conflit (Abramson 1987; Chandler 2017; Bousquet 2018).

Si cette évaluation est exacte pour le contexte de guerre, elle néglige toutefois de prendre en compte le développement de longue durée des systèmes télévisuels militaires. Ceux-ci possèdent en effet plusieurs caractéristiques qui les rendent suffisamment flexibles pour être transférés sans difficulté du miliaire au civil. Tout d'abord, le système télévisuel militaire est distinct, voire fondamentalement différent, d'un système commercial: il ne diffuse pas d'un émetteur à de multiples foyers où se réunit un public de masse mais constitue au contraire un circuit fermé au sein duquel les données (audio-)visuelles sont transférées de la caméra à un seul récepteur. Ensuite, afin d'être intégré à un engin volant et de décoller, le circuit fermé doit être petit et léger. La taille et le poids, mais aussi la simplicité d'usage et de maintenance, sont en effet des critères primordiaux pour de tels systèmes, notamment car le personnel de l'armée n'est pas formé pour manipuler des instruments techniques faillibles ou sophistiqués (Trainer \& Poch 1946).

Cette double caractéristique - fonctionner en circuit fermé et être de taille et de poids réduits, en plus d'être simples d'utilisation - constitue la spécificité matérielle des télévisions militaires qui contribue à leur succès au-delà de leur domaine d'émergence. Dès 1942, en effet, des applications militaro-industrielles sont testées, tout d'abord sur le site Hanford du Manhattan Project, où la télévision en circuit fermé est destinée à faciliter la manipulation de matériel radioactif (Abramson 2003: 10). Quatre années plus tard, la télévision est utilisée dans le cadre des tests de la bombe atomique réalisée dans l'atoll de Bikini. Selon deux ingénieurs de la RCA, ces expériences prouvent 
qu'il existe "un important champ d'utilisation pour la télévision autre que le divertissement» (Barrett \& Goodman 1947: 120). À l'issue de la guerre, la RCA peut dès lors imaginer sans trop de difficultés que la télévision devienne «des yeux dans les usines" qui constitueraient un "moyen complètement nouveau pour [...] contrôler à distance des processus de manufacture [...] autrement inaccessibles ou trop dangereux pour l'homme" (RCA Laboratories News 1946 : s.p.).

Le transfert du circuit fermé militaire vers le secteur industriel se concrétise réellement durant les années 1950. Promues sous le label de "télévision industrielle» ou de "télévision en circuit fermé", les applications industrielles ressemblent beaucoup aux systèmes militaires. Elles sont basées sur un équipement télévisuel de taille réduite et de qualité limitée, et conçues en tant que système autonome: «Léquipement est petit et compact et, en raison de sa simplicité, ne nécessite pas de personnel hautement qualifié.» (Barrett \& Goodman 1947: 124) Grâce à sa simplicité, la télévision industrielle est jugée "versatile», capable de s'adapter à des usages multiples. Sur le plan technologique, elle est d'ailleurs directement tributaire des développements réalisés durant la guerre. Les tubes cathodiques utilisés s'appuient par exemple sur des perfectionnements qui répondaient à l'origine à des objectifs militaires (Barrett \& Goodman 1947:120).

Aux yeux de ses développeurs - parmi lesquels nous retrouvons Zworykin -, la télévision industrielle apparaît comme un système quasi miraculeux :

"Chaque fois que quelque chose est trop dangereux, trop difficile, trop cher, trop incommode, trop inaccessible, trop fatigant, trop éloigné, trop chaud, trop froid, trop haut, trop bas, trop sombre, trop petit pour l'observation directe, utilisez la télévision." (Zworykin et al. 1958: 22)

Pour les auteurs, parce qu'elle offre la vision à distance, la télévision industrielle s'emploie dans tous les contextes où il est préférable de rester à l'écart. Parmi les applications pratiques du dispositif se trouve le contrôle du trafic de route où la télévision permet notamment d'observer le flux des automobiles et de surveiller les feux de circulations qu'il est alors possible de réguler à distance (Kammerer 2009). Selon la municipalité de la ville de Lausanne, qui réfléchit au début des années 1960 à l’installation d'un système télévisuel pour contrôler les artères principales du centre-ville, « ce système a [...] l'avantage incontestable de diminuer l'effectif du personnel qui doit s'occuper de la régulation du trafic» (Conseil communal 1962: 655). 
Intégrée au milieu industriel, la télévision en circuit fermé participe ainsi à rationaliser des tâches répétitives, dangereuses ou autrement pénibles. Dans le contexte de l'après-guerre fortement marqué par l'automatisation puis l'informatisation des processus industriels et administratifs, elle est conçue comme un dispositif de l'efficacité et de l'optimisation. Comme le résume une publicité française au milieu des années 1950, elle substitue "à l'homme une machine, aussi ou même plus intelligente que lui " ${ }^{6}$. Que ce soit sur le champ de bataille ou dans les halles de production, la télévision promet la possibilité de se défaire de l'homme. Pour résumer, l’imaginaire du unmanning détermine autant la recherche militaire que les applications civiles.

\section{Les images opérationnelles de la télévision}

Comme nous l'avons souligné, la substitution de l'homme par la machine dans le domaine industriel ne se réduit pas à un champ particulier. Dès les années 1950, la presse et les publications spécialisées américaines et européennes reprennent et amplifient l'idée d'étendre les applications de la télévision à une multitude de secteurs. Ils destinent alors la télévision industrielle à s'insérer littéralement dans tous les espaces et à réaliser toutes les tâches possibles et imaginables. Que ce soit dans les aéroports ou les parcs d'attractions, dans les auditoires universitaires ou les centrales nucléaires, les circuits fermés de télévision doivent permettre le contrôle de la production, la surveillance, la manipulation de matériel à distance, l'observation de matériaux dangereux, etc. La télévision industrielle promet dès lors de devenir « un outil de base pour un nombre toujours croissant d'opérations futures, aussi bien commerciales qu'industrielles, éducatives et gouvernementales " (Noll 1956:1). Selon un journaliste suisse qui a assisté à une démonstration à Zurich réalisée par une entreprise britannique, la télévision industrielle est «Bonne à tout faire» :

"La nouvelle caméra de télévision industrielle se distingue par ses dimensions réduites et pratiques qui en font un appareil universel. [...] Les possibilités d'emploi de la télévision industrielle moderne sont pratiquement illimitées. [...] Il ne sera pas difficile de trouver à l'avenir d'autres domaines où la télévision industrielle pourra être utilisée avec succès. Elle deviendra de plus en plus une "bonne à tout faire" dont l'avenir s'annonce particulièrement brillant." (Journal d'Yverdon 1955)

\footnotetext{
6 Publicité française de 1957, disponible sur https://www.periodpaper.com/collections/phono-radio-tv/ products/1957-ad-lautomatic-industrial-television-123-boulevard-massena-paris-french-174959-ven7-116, consulté le $1^{\mathrm{er}}$ octobre 2019.
} 
Comme en témoigne cet article suisse, la télévision industrielle n'est pas uniquement développée dans les laboratoires de la RCA: elle constitue un phénomène largement transnational qui se perfectionne en parallèle de la mise en place des services respectivement publics en Europe et commerciaux aux États-Unis. Les discours sur la télévision industrielle se ressemblent par leur enthousiasme pour cette application télévisuelle et par leur célébration de l'automation et de la rationalisation qu'elle augure. Ils promeuvent par ailleurs la télévision industrielle comme un "média universel».

L’universalité de la télévision en circuit fermé découle certes de son adaptabilité, mais également de son statut d'instrument plutôt que de moyen de communication. La télévision industrielle est un "outil de base" (Noll 1956) et une "bonne à tout faire» (Journal d'Yverdon 1955). Facilitant des procédures industrielles et militaires, elle est moins médiatrice dans un échange communicationnel qu'opératrice au sein d'un processus qui tend vers l'automatisation.

Dans son travail sur les images de la guerre du Golfe, Farocki a justement proposé la notion d'«images opérationnelles" (operative Bilder) pour définir les images qui "font» quelque chose, plutôt qu'elles ne montrent le monde. Pour Farocki, les images opérationnelles "ne représentent pas un objet» mais se caractérisent par leur potentiel agissant (Farocki 2004). Selon l'historien du cinéma Thomas Elsaesser, l’image opérationnelle de Farocki est une "image qui fonctionne comme instruction à une action" (Elsaesser 2014). Aujourd'hui, la notion d'images opérationnelles est mobilisée dans le cadre des Digital Media Studies où elle sert à décrire les nouvelles caractéristiques de l'image numérique (Hoel 2018). Intégrée dans un dispositif informatique, l’image est augmentée par des métadonnées et préparée à être lue par l'ordinateur via les processus de l'intelligence artificielle et de la machine vision.

À l’instar des algorithmes numériques qui demeurent tributaires des interactions homme-machine, l'automation totale de la production et de l'interprétation visuelle n'est pas réalisée dans le cas des images du circuit fermé télévisuel. L'effort d’évincer l'homme par des discours et des représentations est surtout le signe du poids de celuici, que ce soit sur le champ de bataille ou dans les halles de production. Souligner l'opérationnalité des images télévisuelles permet toutefois de cerner une parenté supplémentaire entre les domaines militaires et civils: dans les exemples cités tout au long de cet article, voir signifie aussi faire - surveiller, commander, tuer. 


\section{Conclusion}

La machine télévisuelle " bonne à tout faire » sera rapidement détrônée par l'ordinateur, autre «machine universelle». Cependant, l’avenir de la télévision en circuit fermé ne sera pas moins radieux: sous forme de la vidéosurveillance des espaces publics et privés, le circuit fermé télévisuel nous accompagne au quotidien depuis les années 1990. Paradigmatique de la surveillance généralisée de nos sociétés, qui se répand aujourd'hui non seulement off-mais également on-line, la vidéosurveillance fait partie de cette histoire élargie de la télévision qui englobe également les premières recherches sur les drones.

Dans la perspective d'une archéologie de la télévision, les recherches menées par la RCA autour des drones et des missiles téléguidés éclairent ainsi d’un jour nouveau l'histoire d'un média hautement élastique. La télévision industrielle, issue de recherches militaires, connaît son essor en parallèle de la conquête des salons familiaux par le mass media télévisuel. En cela, elle nous oblige à penser le média au-delà du paradigme d'«information, d'éducation, et de divertissement» qui caractérise l'idéal du service public européen. À partir de ce constat, la notion d'«images opérationnelles " offre un cadre épistémologique efficace pour appréhender la spécificité d'un dispositif télévisuel qui est davantage instrument que moyen de communication.

Dans la perspective d'une analyse de la guerre des drones, le détour par la télévision militaire ouvre plusieurs pistes qui dépassent la seule histoire technico-militaire du perfectionnement des engins sans équipages. Le passage par les laboratoires de la RCA livre non seulement des informations sur les premiers programmes de recherche sur les drones aux États-Unis, mais révèle surtout la facilité avec laquelle la recherche militaire est transférée vers le civil. La télévision militaire, renommée télévision industrielle après la guerre et déplacée des avions téléguidés dans les halles de production industrielle, témoigne avec acuité des perméabilités entre usages, technologies et politiques. Si la circulation des technologies du militaire au civil est bien connue pour l'informatique ou le nucléaire, les débats actuels sur les drones postulent très souvent implicitement une séparation nette entre ces domaines: l'utilisation du drone civile serait à mille lieues des usages armés. Une analyse historique portant sur l'émergence des aéronefs sans équipage va à l'encontre d'un tel discours rapide. Elle permet au contraire de nourrir les recherches d'auteur.e.s qui soulignent l'impossible distinction entre les espaces militaires et civils et qui examinent le rôle joué par le drone dans l'imbrication actuelle de ces espaces (Neocleous 2013; Bracken-Roche 2016; Kaplan 2017; Parks 2018 et Parks dans ce numéro). Une telle étude historique permet 
plus généralement de nuancer les fantasmes et projections actuelles quant aux utilisations foisonnantes des drones, et d'éclairer les prémices technologiques et idéologiques du «progrès » contemporain.

\section{Remerciements}

Je tiens à remercier chaleureusement Marie Sandoz, Claire-Lise Debluë et Mathilde Zbaeren pour leurs relectures attentives et leurs commentaires précieux. La recherche pour cet article a bénéficié du soutien du Fonds national suisse pour la recherche scientifique (FNS) qui m'a octroyé une bourse Early Postdoc.Mobility pour mon projet postdoctoral A Media Archaeology of Drones: Television in the Military, 1930s-1940s (P2LAP1_174743). 


\section{Références}

Abramson Albert (1987), The History of Television, 1880 to 1941. Jefferson, McFarland.

Abramson Albert (2003), The History of Television, 1942 to 2000, Jefferson, McFarland.

ANONYME, "Radio Research in Wartime», document non daté et non signé du fonds R. Kenyon Kilbon, boîte 1108, fourre 58, David Sarnoff Research Center records (Accession 2464.09), Hagley Museum and Library, Wilmington, DE 19807.

Anonyme (1946), "Airborne Television Shown at Joint Navy-RCA Demonstration ", RCA Laboratories News, vol. 3, $\mathrm{n}^{\text {os }} 2$ \& 3, s.p.

96 ANONYME (1955), "La télévision industrielle. Bonne à tout faire ", Journal d'Yverdon, 5 novembre 1955 .

BARrett R. E. \& Goodman M. M. (1947), "Simplified Television for Industry», Electronics, juin 1947, pp. 120-124.

Bousquet Antoine (2018), The Eye of War. Military Perception from the Telescope to the Drone, Minneapolis/Londres, University of Minnesota Press.

Bracken-Roche Ciara (2016), "Domestic Drones: the Politics of Verticality and the Surveillance Industrial Complex", Geographica Helvetica, nº 71, pp. 167-172.

Burns Russel W. (1998), Television: An International History of the Formative Years, Londres, Institution of Electrical Engineers.

Снам аyou Grégoire (2013), Théorie du drone, Paris, La Fabrique.

CHANDle R Katherine (2017), "American Kamikazes: Television-Guided Assault Drones in World War II", in Life in the Age of Drone Warfare, L. Parks et C. Kaplan (dir.), Durham/Londres, Duke University Press, pp. 89-111.

CH ANDle R Katherine (2020), Unmanning. How Humans, Machines and Media Perform Drone Warfare, New Brunswick, Rutgers.

Conseil communal de Lausanne (1962), Séance du mardi 26 juin, «Signalisation lumineuse automatique», Préavis $n^{\circ} 37$, pp. 652-657.

EDWARds Paul N. (1996), The Closed World: Computers and the Politics of Discourse in Cold War America, Cambridge, MIT Press.

Elsaesser Thomas \& Alberro Alexander (2014), "Farocki: A Frame for the No Longer Visible: Thomas Elsaesser in Conversation with Alexander Alberro ", e-flux, $\mathrm{n}^{\circ}$ 59. URL: https://www.e-flux.com/journal/59/61111/farocki-a-frame-for-the-no-longer-visible-thomas-elsaesser-in-conversation-with-alexander-alberro/, consulté le 15 octobre 2019.

Everett H. R. (2015), Unmanned Systems of World Wars I and II, Cambridge, MIT Press.

FAHRney Delmar S. (1980), "The Birth of Guided Missiles", Proceedings of the United States Naval Institute, $\mathrm{n}^{\circ} 106, \mathrm{pp} .54-60$. 
FARocki Harun (2004), «Phantom Images », Public. Art, Culture, Ideas, nº 29, pp. 13-22.

Fickers Andreas \& Weber Anne-Katrin (2015), "Editorial: Towards an Archaeology of Television ", VIEW Journal of European Television History and Culture, vol. 4, $\mathrm{n}^{\circ}$ 7, pp. 1-7.

HiPPLE R Thomas (2014), Le gouvernement du ciel: Histoire globale des bombardements aériens, Paris, Les Prairies ordinaires.

Hoel Aud Sissel (2018), "Operative Images: Inroads to a New Paradigm of Media Theory", in Image - Action - Space: Situating the Screen in Visual Practice, L. Feiersinger, K. Friedrich et M. Queisner (dir.), De Gruyter, pp. 11-27.

How ETH Linwood S. (1963), History of Communications. Electronics in the United States Navy, Washington, U.S. Government Printing Office.

Kammerer Dietmar (2009), "Police Use of Public Video Surveillance in Germany 1956: Management of Traffic, Repression of Flows, Persuasion of Offenders ", Surveillance \& Society $6, \mathrm{n}^{\circ} 1$, pp. 43-47.

KAPLAN Caren (2017), "Drone-O-Rama: Troubling the Temporal and Spatial Logics of Distance Warfare", in Life in the Age of Drone Warfare, L. Parks et C. Kaplan (dir.), Durham/Londres, Duke University Press, pp. 161-177.

Keilbach Judith \& Stauff Markus (2013), "When Old Media Never Stopped Being New. Television's History as an Ongoing Experiment", in After the Break. Television Theory Today, M. de Valck et J. Teurlings (dir.), Amsterdam, Amsterdam University Press, pp. 79-98.

NDRC (National Defense Research Committee) (1946), Summary Technical Report of Division 5, Vol 1: Guided Missiles and Techniques, Washington.

Neocleous Mark (2013), "Air Power as Police Power ", Environment and Planning D: Society and Space, vol. 31, pp. 578-593.

Noll Edward M. (1956), Closed-Circuit and Industrial Television, New York, Macmillan.

Packer Jeremy \& Reeves Josh (2013), "Romancing the Drone: Military Desire and Anthropophobia from SAGE to Swarm ", Canadian Journal of Communication, vol. 38, n 3. URL: https://doi.org/10.22230/cjc.2013v38n3a2681, consulté le $1^{\text {er }}$ octobre 2019.

PANTENBURG Volker (2017), "Working Images: Harun Farocki and the Operational Image ", in Image Operations. Visual Media and Political Conflict, J. Eder et C. Klonk (dir.), Manchester, Manchester University Press, pp. 49-62.

PARIKKA Jussi (2012), What Is Media Archaeology, Polity.

PARKS Lisa (2018), Rethinking Media Coverage. Vertical Mediation and the War on Terror, New York/Londres, Routledge.

RoE John H. (1950 [1947]), " New Television Field-Pickup Equipment Employing the Image Orthicon", in Television. Volume V (1947-1948), A. Goldsmith, A. Van Dyck, R. Burnap, E. Dickey et G. Baker (dir.), Princeton, RCA, pp. 1-30. 
Rollins Peter C. (1992), "Introduction: The Gulf War and Television », Film \& History: An Interdisciplinary Journal of Film and Television Studies, vol. 22, $\mathrm{n}^{\circ \mathrm{s}} 1$ \& 2, pp. 1-10

SARnof F David (1946), "Introduction to Technical Papers on Airborne Television", RCA Review. A Technical Journal, vol. 7, $\mathrm{n}^{\circ} 3$ (septembre), pp. 291-292.

Trainer M. A. \& Poch W. J. (1946), "Television Equipment for Aircraft», RCA Review. A Technical Paper, vol. 7, $\mathrm{n}^{\circ} 4$ (décembre), pp. 469-502.

URICchio William (2009), "Télévision: l’institutionnalisation de l’intermédialité», in La télévision du téléphonoscope à Youtube : pour une archéologie de l'audiovision, M. Berton et A.-K. Weber, Lausanne, Antipodes, pp. 161-179.

WEBER Anne-Katrin (à paraître), Interwar Television on Display. New Media and Exhibi98 tion Culture in Europe and the USA, 1928-1939, Amsterdam, Amsterdam University Press. Zubeldia Océane (2012), Histoire des drones, Paris, Perrin.

ZWORY KIN Vladimir K. (1946), «Flying Torpedo With an Electric Eye», RCA Review. A Technical Journal, vol. $7, \mathrm{n}^{\circ}$ 3, pp. 293-302.

ZWORY Kin Vladimir K., RAMBERG E. G. \& Flory L. E. (1958), Television in Science and Industry, New York, John Wiley \& Sons. 